\title{
Comparison of different configurations of Phase Doppler Analyser
}

\author{
Matouš Zaremba ${ }^{1, a}$, Milan Malý ${ }^{1}$, Jan Jedelský ${ }^{1}$ and Miroslav Jícha ${ }^{1}$ \\ ${ }^{1}$ Brno University of Technology, Faculty of Mechanical Engineering, Department of the thermodynamics and environmental engineering, \\ Technická 2896/2, 61669 Brno, Czech Republic
}

\begin{abstract}
A phase Doppler anemometry (PDA) technique is widely used in experimental fluid mechanics to measure size and velocity of particles in the fluid flow. Even though this method is common in experimental fluid mechanics, there are only few techniques that might serve for the purpose of the evaluation of the PDA system. To examine results of the PDA visualizations techniques are usually used. However, this approach suffers from several aspects. Mainly, it is difficult to determine the exact position of the measurement volume of PDA system. Then it is complicated to determine which particles are passing through the measuring volume. Another way how to examine performance of the PDA system is to use two PDA systems simultaneously. By using one laser for both systems we can avoid previously mentioned aspects. In our experiments, we use fiber based PDA system and classical PDA system both made by Dantec Dynamics. The aim of this paper is to compare results from various configurations and highlight crucial parameters that influence measurements.
\end{abstract}

\section{Introduction}

Non-intrusive optical measuring techniques play an important role in the field of the experimental fluid mechanics. Especially, in the application of measuring velocity and size of droplets or bubbles in two-phase flows. These parameters are of importance in several aspects in industrial applications. Mainly in the field of spray combustion systems such as automotive and aircraft engines, combustion of liquid fuels in power plants or spray drying processes in the food industry [1].

Several techniques were developed to measure characteristics of the spray, for example: particle image velocimetry (PIV), laser Doppler anemometry (LDA), phase Doppler anemometry (PDA), Malvern laser particle size analyser and others. These measuring techniques give us valuable and relatively precise results about droplet velocity or droplet size or both. However, determining the uncertainty of the measuring system is a difficult task $[2,3]$. Even though several workers tried to calculate precision of PDA system a precise method has not yet been made. Some publications investigated different measuring techniques, for example: [4] however, the comparison between two system which works on the same principle but have different post processing tools or different configuration was examined in just few publications, for example [5]. Moreover, various commercial PDA systems were developed in the past, and their performance is usually assumed to be precise. It is common to compare results from measurements with previous observations. However, the difference between measuring systems should be taken into account. Thus, in this paper we focus on the

\footnotetext{
${ }^{\mathrm{a}}$ Corresponding author: zaremba@fme.vutbr.cz
}

examination of two different phase Doppler analysers in three configurations. The aim is to find crucial parameters or components of the PDA systems that influence measured results.

\section{Material and Methods}

Experiments were conducted on the cold test bench at still ambient condition and room temperature at the Brno University of Technology in Sprays laboratory.

\subsection{Atomizer}

The PDA systems were tested in the fully developed spray. The spray was generated by the twin-fluid atomizer, so called outside-in liquid injection atomizer (OIL). This type of atomizer works on a principle of injecting liquid and gas into the mixing chamber where two-phase flow is created. Then this mixture is pushed downstream the atomizer and through the exit orifice into the ambient atmosphere. Detailed description of the atomizer can be found in [6]. Description of the test bench can be found in [7]. Light heating oil (LHO) and pressurized air were used as operating fluids. Physical properties of these fluids are presented in Table 1.

Operating regime of the atomizer is determined by the operating overpressure inside the atomizer and liquid and gas mass flows. These parameters were captured during the experiment. Measured values are written in the Table 2. Values of mass flows result in gas to liquid ratio (GLR) of $20 \%$ which indicates the annular two-phase flow inside the atomizer [6]. 
Table 1. Physical properties of used fluids.

\begin{tabular}{|c|c|c|c|}
\hline Name & $\begin{array}{c}\text { Dynamics } \\
\text { viscosity } \\
(\mathbf{k g} /(\mathbf{m} \cdot \mathbf{s}))\end{array}$ & $\begin{array}{c}\text { Density } \\
\left(\mathbf{k g} / \mathbf{m}^{3}\right)\end{array}$ & $\begin{array}{c}\text { Surface } \\
\text { tension } \\
\left(\mathbf{k g} / \mathbf{s}^{\mathbf{2}}\right)\end{array}$ \\
\hline LHO & 0.0185 & 874 & 0.0297 \\
\hline Air & $1.82 \cdot 10^{-5}$ & 1.23 & - \\
\hline
\end{tabular}

Table 2. Operating regime of the atomizer.

\begin{tabular}{|c|c|c|c|}
\hline $\begin{array}{c}\text { LHO mass } \\
\text { flow } \\
(\mathbf{k g} / \mathbf{h})\end{array}$ & $\begin{array}{c}\text { LHO } \\
\text { pressure } \\
\mathbf{( k P a )}\end{array}$ & $\begin{array}{c}\text { Air mass } \\
\text { flow } \\
\mathbf{( k g / h )}\end{array}$ & $\begin{array}{c}\text { Air pressure } \\
\mathbf{( k P a )}\end{array}$ \\
\hline 2.55 & 66 & 0.13 & 69 \\
\hline
\end{tabular}

\subsection{Description of examined systems}

In this work, we examined two phase Doppler analysers in three configurations. The first configuration (system A) is fiber based 2D phase Doppler analyser made by Dantec Dynamics. This system consists of:

- Spectra Physics Stabilite 2017 Argon laser: max. power output 6 Watts,

- 60X41 Transmitter, which splits the beam into its individual color components $(488.0 \mathrm{~nm}, 514.5 \mathrm{~nm})$ and divides each color into two beams.

- Brag cell is implemented in the transmitter which gives frequency shift $40 \mathrm{MHz}$ to each beam from given pair.

- 60X81 2D $85 \mathrm{~mm}$ transmitting optics with 50X82 beam translator and focal length of the final lens of $500 \mathrm{~mm}$,

- 57X50 $112 \mathrm{~mm}$ diameter fiber PDA receiver optics with spatial filter and focal length of the lens of 800 $\mathrm{mm}$,

- Fiber PDA Detector unit and

- BSA P80 flow and particle processor that was set to measure velocity within the range from 0 to $25 \mathrm{~m} / \mathrm{s}$ and droplet size in the range from 0 to 274 microns.

The transmitting line (laser, transmitter and transmitting optics) of the system A was used for all measurements to keep the same parameters of measuring volume (m.v.). The second configuration (system B) is classic 1D Dantec phase/Doppler particle analyser. Transmitting optics and processor were used from the system A. So the receiving line of the system B consists of:

- Dantec 57X10 receiving optics with focal length of a final lens of $500 \mathrm{~mm}$ and

- BSA P80 flow and particle processor.

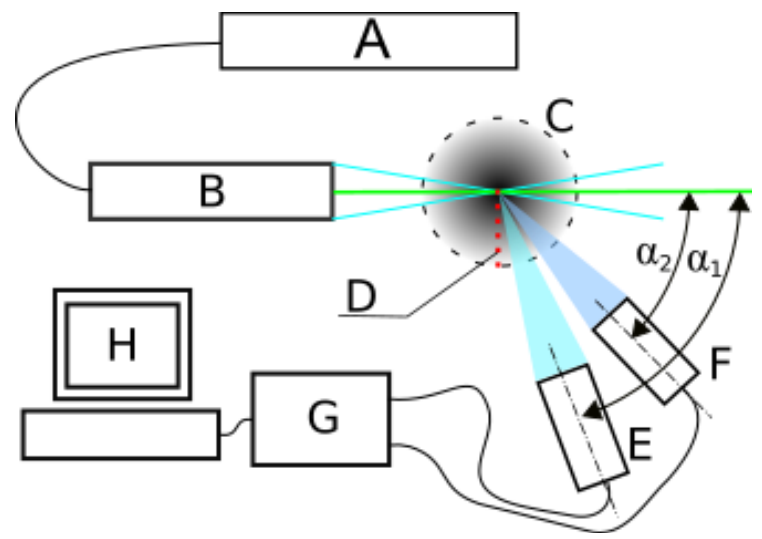

Figure 1. Schematic layout of PDA system. A - Laser, B transmitting optics, C - Spray, D - measured points, E Fiber PDA receiving optics, $\mathrm{F}$ - classic PDA receiving optics, $\mathrm{G}$ - processor, $\mathrm{H}$ - computer.

Third configuration (system C) uses again transmitting line from the system A but with the processor from classic PDA system. Then, the receiving line of system $\mathrm{C}$ consists of:

- Dantec 57X10 receiving optics with focal length of a final lens of $500 \mathrm{~mm}$ and

- Dantec Model 58N50 signal processor that was set to measure velocity within the range from 0 to 25 $\mathrm{m} / \mathrm{s}$ and droplet size in the range from 0 to 236 microns.

\subsubsection{Parameters of the measuring volume}

Two intersecting laser beams coming out from the transmitting optics create light objects in the intersecting point, so-called measuring volume (m.v.). This ellipsoid consists of layers of high and low intensities of light i.e. fringes. The optical configuration of examined system results in fringe separation of 2.14 $\mu \mathrm{m}$ and dimensions of m.v. $0.08 \times 0.08 \times 0.7 \mathrm{~mm}$.

The effective volume of m.v. has a shape of an ellipsoid truncated by a slit shaped by a spatial filter placed in the receiver optics [8] as shown in the figure 2. We assume that m.v. is symmetric among the main axis. Effective length of the m.v. is determined by the slit used in the receiving optics. In system $\mathrm{A}$, the slit was set to $200 \mu \mathrm{m}$ which results into the length $\mathrm{L}_{\mathrm{S}}=0.52 \mathrm{~mm}$. The slit of systems B and C result in effective m.v. length $\mathrm{L}_{\mathrm{S}}=0.26 \mathrm{~mm}$.

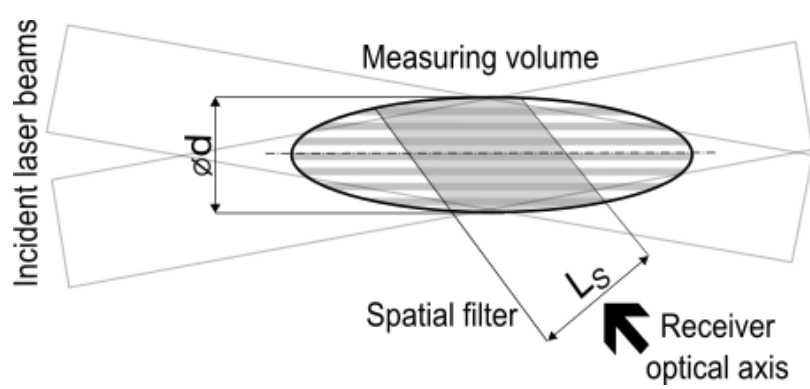

Figure 2. Measuring volume geometry. 


\subsubsection{Experimental setup}

Measurements were performed in the axial distance of $100 \mathrm{~mm}$ from the atomizer's exit orifice. This distance was chosen because we predict that only spherical droplet should be presented in this area according to our previous findings $[9,10]$. We also calculated Weber number (We) of droplets, and we found out that $97.5 \%$ of droplets have lower We than the critical value (11 in our case [1]). The mean value of We is then equal to 2, so we assume that all atomization processes are finished. Thus, PDA system, which can measure only spherical droplets, is able to measure with high validation $(70-90 \%$ depends on the position in the spray). Due to the symmetry of the spray we measured only one half of the radial profile: 5 points by the step of $5 \mathrm{~mm}$. Systems were set to measure 50000 samples or one minute in low dense regions. Only axial velocity component was measured due to the fact that systems B and C are 1D only.

The PDA setup was optimized to a given spray conditions to get the highest validation and data rate. The velocity and drop size measuring spans were set according to preliminary measurements to be able to detect all droplet sizes and velocities. Systems B and C were set to get the similar velocity and droplet size measuring spans as it was in the system A. However due to different construction of PDA receiving optics the exact same values cannot be reached.

The PDA receiving optics is ideally set into the Brewster's angle where only one scattering mode is dominant. In our case, it is 69 degrees to the main axes of transmitting optics (shown as angle $\alpha$ in figure 1). Due to limited optical access to the spray, consequently to the m.v., systems were set into two different scattering angles. System A was put in the angle of $\alpha_{1}=75$ degrees and systems $B$ and $C$ were set in the angle of $\alpha_{2}=65$ degrees, see figure 1 . The difference in measured values from various scattering angles was checked before the measurements. Variations in the mean axial velocity were in the range of $1.5 \%$ from measured range. Mean diameter differences were in the range of $1.4 \%$. Thus, we assume that deviation from the ideal scattering angle has only negligible influence on our results.

\subsubsection{Repeatability of the measurements}

For this purpose only system A was used in a standard configuration as mentioned above. Measurements were performed in the same positions in the spray and results are plotted in graphs as error bars showing measured span of values. These results serve for the purpose of prediction of an error caused by the repetition of measurements.

\section{Results and discussion}

As the first examination of the measurements, we investigate velocity and drop size profiles. The upper graph in figure 4 shows axial velocity profiles with error bars obtained by the repetitive measurements and typical data rate profile.
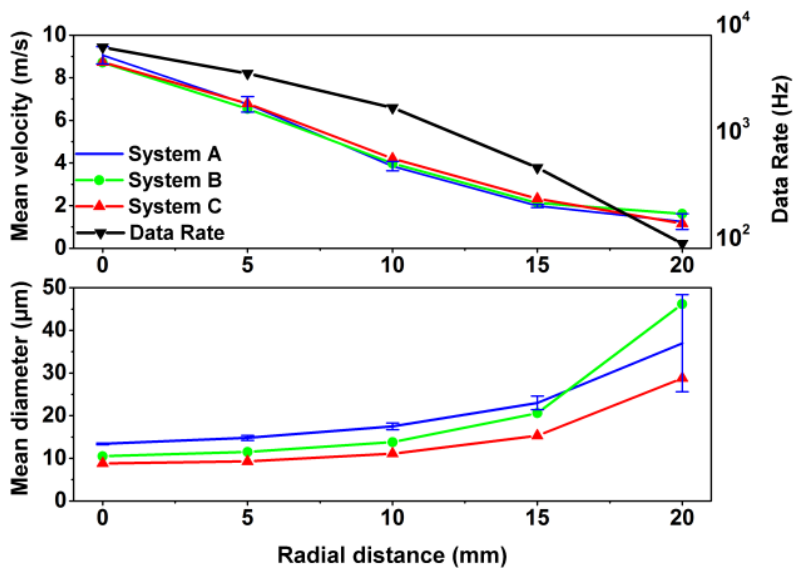

Figure 3. Mean axial velocity and mean diameter profiles. The zero position corresponds to the centre of the spray.

The differences between velocities are in the range from minimum $3.7 \%$ to maximum of $22 \%$ from a measured value which are $1 \%$ and $1.8 \%$ from measured range. Minimal difference value was found in the radial distance of $5 \mathrm{~mm}$ and maximal in the distance of $20 \mathrm{~mm}$. The maximal difference is probably caused by a low data rate (see figure 3 ) and by an instability of the spray at the edge [11]. Nevertheless, considering error bars we see that the majority of measured points fall into the error range. Thus, we assume that difference in mean velocity might be caused by the uncertainty of repetitive measurements. We assume that systems were set correctly and further in this paper we focus on an examination of one point only (in the centre of the spray).

Drop size distribution profiles are shown in figure 3 at the bottom. It indicates that there is a demonstrable influence of the receiving optics and used processor to the resulting drop size distribution. The system A measured largest droplets except of value at the edge of the spray. The system B measured smaller droplets and the system A the smallest droplets. Only one point in a profile falls into predicted range of the error. This point is at the edge of the spray where the largest error was observed. We assume that this behaviour was caused again by low data rate and instabilities of the spray.

One of the ways how to examine PDA data it is to plot transit time (TT) of droplets through the measuring volume versus droplet size or velocity. This approach was recommended in PDA system manuals [12]. Ideally, when droplet flows through the centre of the m.v. magnitude of TT should be linearly dependent on droplet size and velocity. When particle is not flowing through the centre of the m.v. then TT would not be directly proportional to the droplet size and velocity. However, as mentioned in [12] linear relationship should be observed for the majority of measured droplets. Thus for evaluation of droplet size measurements we plot diameter of droplets versus TT, see figure 4 .

Results in figure 4 show that systems A and B perform similarly. However, system $\mathrm{C}$ somehow rounds the values of TT. This is an effect of maximal sampling 
frequency. Systems were set to have the highest measuring resolution. Minimal TT that can be measured for systems A and $\mathrm{B}$ was $0.1 \mu \mathrm{s}$ and for the system $\mathrm{C}$ was $2 \mu \mathrm{s}$. Thus, the fringe pattern, presented in figure 4 for the system $\mathrm{C}$, is a consequence of its resolution.

Relationship of TT and droplet size show that droplet size is relatively well correlated with TT for most particles. However, there are large droplets presented in the low values of TT (mainly in the cases of system A and B) and small ones in the range of high TT (system C). It means that those large particles flew probably on the edge of measuring volume. Assuming that laser beams have Gaussian intensity profile then Gaussian beam effect might occur [13]. This effect causes bias in PDA measurements. There is an approach how to distinguish false particles generated by this effect [14]. This approach uses signal amplitude analysis which is not possible in our systems.

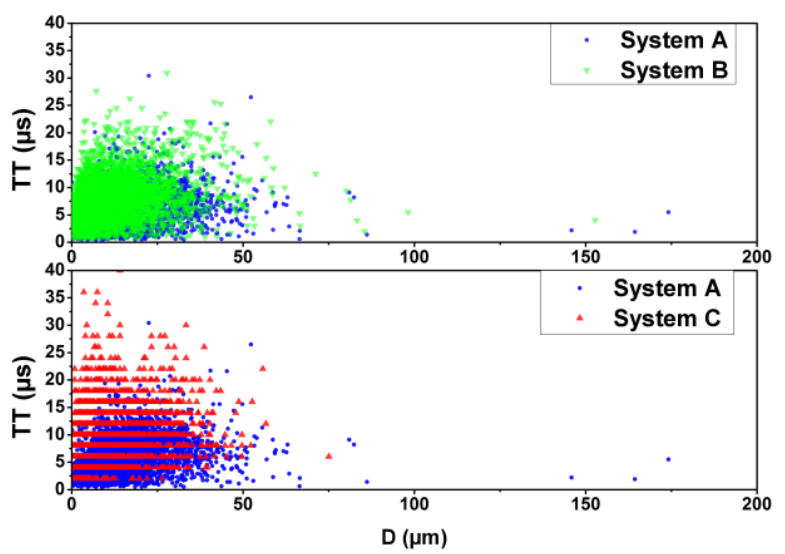

Figure 4. Droplet size-transit time correlation. Only 5000 samples are shown for better visibility.

When examining small droplets we calculated ideal TT from a given velocity, the diameter of m.v. and droplet diameter. Ideal TT in this case means that we predict a droplet which flew through the centre of the m.v. We observed that $99 \%$ of small particles with TT higher than $25 \mu \mathrm{s}$ had higher measured TT than computed idealised value. It means that they travelled longer trajectory in a given time than is a diameter of the m.v.. Thus, we assume that small particles with the TT should not be real. However, our approach is simplified and we do not take into account variations in signal to noise ratio, the precision of the laser beam alignment, changes in droplet velocity and other factors.

\section{Conclusions}

We examined two PDA systems in three configurations in this paper. Results show that under the same conditions these systems measure velocity precisely, but values of mean droplet diameter are different. Variations in droplet size and TT were found for the system C where 58N50 processor was used. Moreover, we observed that systems $\mathrm{A}$ and $\mathrm{B}$ measure more large particles (larger than 100 microns) whereas the system $\mathrm{C}$ measures more small particles that have very small transit time.
Thus, caution has to be made when comparing results from other measuring systems.

An approach, recommended by the manufacturer, points to the fact that probably several invalid particles were measured. However, the method presented in this paper is not precise enough, and it should be extended by evaluation of signal amplitude, or it should be supported by the high-speed imaging of the m.v. during the measurements. This, however, requires self-made PDA system or camera with long distance microscope synchronized with PDA system.

\section{Acknowledgements}

The presented research was supported by project No. GA15-09040S, which was funded by the Czech Grant Agency, the project LO1202 NETME CENTRE PLUS with the financial support from the Ministry of Education, Youth and Sports of the Czech Republic under the "National Sustainability Programme I" and project Reg. No. FSI-S-14-2355 funded by the Brno University of Technology.

\section{References}

1. Lefebvre, A., Edtion ed.: Taylor \& Francis, ISBN 9780891166030, (1988)

2. Calvo, E., J. Garcia, I. Garcia and L. Aisa Experiments in Fluids, 47(3), 489-508, (2009)

3. Czarske, J. W. Optics Letters, 21(7), 522-524, (1996)

4. Dullenkopf, K., M. Willmann, S. Wittig, F. Schone, et al., Particle \& Particle Systems Characterization, 15(2), 81-89, (1998)

5. Wigley, G., J. Heath, G. Pitcher and A. Whybrew, Particle and Particle Systems Characterization, 18(4), 169-178, (2001)

6. Mlkvik, M., P. Stahle, H. P. Schuchmann, V. Gaukel, et al. International Journal of Multiphase Flow, 77, 19-31, (2015)

7. Jedelský, J., M. Zaremba, M. Malý and M. Jícha., EPJ Web of Conferences, 67, (2014)

8. Albrecht, H. E., N. Damaschke, M. Borys and C. Tropea, Springer Berlin Heidelberg, ISBN 9783662051658, (2013)

9. Jedelský, J., M. Jícha, J. Sláma and J. Otáhal Energy \& Fuels, 23, 6121-6130, (2009)

10. Jedelský, J. and M. Jícha. In WIT Transactions on Engineering Sciences. 82, 227-238, (2014)

11. Jedelský, J. and M. Jícha, Atomization and Sprays, 18(1), 49-83, (2008)

12. Dantec Dynamics, Edtion ed. Tonsbakken, 18, DK2740 Skovlunde, Denmark, 119-122, (2001)

13. Albreacht, H., M. Borys, M. Wenzel and T. Wriedt, Particle \& Particle Systems Characterization, 11(4), 339-344, (1994)

14. Bachalo, W. D., patent, US4986659 A, (1991). 